\section{PDT-Pflaster zur Behandlung von aktinischen Keratosen}

Dermasence übernimmt ab sofort den exklusiven Vertrieb der Alacare ${ }^{\oplus}$ PDT-Pflaster in Deutschland und Österreich. Das Alacare $^{\circledast}$ PDT-Pflaster wird für die Behandlung aktinischer Keratosen auf der Kopfhaut oder im Gesicht angewendet. Die photodynamische Therapie erfolgt damit in zwei Schritten. Nachdem der Arzt das Pflaster verordnet hat, beginnt der erste Schritt mit der Applikation. Hierbei werden die Pflaster von geschul- tem Fachpersonal im Beisein des Arztes auf die betroffenen Stellen aufgeklebt.

Nach einer vierstündigen Einwirkzeit, in der der Patient die Praxis verlassen kann, erfolgt der zweite Schritt: „Beleuchtung der behandelten Läsionen“. Hier werden die Stellen für einige Minuten mit Rotlicht bestrahlt. Die Bestrahlung löst in den Zellen der veränderten Hautstellen eine chemische Reaktion aus, wodurch die geschädigten Hautzellen zerstört werden. Jedes Pflaster enthält $8 \mathrm{mg}$ 5-Aminolävulinsäure zur Verabreichung einer genau definierten Wirkstoffmenge. Bis zu 6 Pflaster können in einer Therapiesitzung appliziert werden.

Zur Einbettung der medizinischen Behandlung unterstützt ein therapiebegleitender Hautpflegeplan. Der Hautpflegeplan bei aktinischen Keratosen wurde zusammen mit Prof. Dr. Szeimies, Klinikum Vest, entwickelt und kann ab sofort bei Ihrem Außendienst abgerufen werden. Für Fragen melden Sie sich gerne auch telefonisch beim Alacare ${ }^{\circledast}$ Innendienst-Ansprechpartner unter der Telefonnummer +49251 91599-220.

Nach Informationen von Dermasence

\title{
Atopische Haut
}

\section{Barrierefunktion der Haut wiederherstellen}

Atopische Haut ist gekennzeichnet durch einen verminderten Feuchtigkeitsgehalt, einen erhöhten transepidermalen Wasserverlust, einen Mangel an FilaggrinProtein mit Beeinträchtigung der epidermalen Schutzfunktion und ein Defizit an Sphingolipiden und Ceramidvorstufen mit natürlichen feuchtigkeitsbindenden und antimikrobiellen Eigenschaften.

Mit Xerodiane AP+ Balsam und Xerodiane AP+ Creme haben die Laboratoires noreva zwei Präparate entwickelt, die den besonderen Bedürfnissen der zu Atopie und zu mikrobiellen Ekzemen neigender Haut gerecht werden. Sie bil- den einen unmerklichen, nicht okklusiven, schützenden und nicht klebenden Film auf der Haut, der ein angenehm samtiges Gefühl hinterlässt.

Auf der Basis von zwei patentierten Wirkstoffkombinationen (Vitamin B3/ Phytosphingosine und Hyaluronsäure/ Silberionen) verbunden mit Zemea (Maiskeimglukose), Omega-3-, -6- und -9-Fettsäuren im hochwertigen IncaInchi-Öl sowie Physio-Filag, das den Mangel an Filaggrin kompensiert, wird die Barrierefunktion der Haut rasch wiederhergestellt, die Wasserspeicherfähigkeit und der Feuchtigkeitsgehalt der Haut erhöht sowie die bakterielle Vermehrung auf der Haut gehemmt. Die Neigung zu Entzündungen und quälendem Juckreiz wird nachhaltig vermindert.

Xerodiane AP+ Balsam und Creme können von den Patienten je nach persönlichem Empfinden oder dem Wunsch nach mehr oder weniger Fett gewählt werden. Sie können auch schon während des Ekzemschubes therapieergänzend verwendet werden. Für die beim atopischen Ekzem besonders betroffenen Hautareale, wie Hals, Ellenbeugen, Kniebeugen und Handgelenke unterstützt Xerodiane $\mathrm{AP}+$ reizmindernde Creme die therapeutischen Maßnahmen mit Glukokortikoiden und kann sie unter Umständen sogar einsparen.

Nach Informationen von Horst Spickermann

\section{Augen-Make-up schonend und wirkungsvoll entfernen}

Die richtige Gesichtsreinigung ist die wichtigste Grundlage, um Hautirritationen und Komedonen vorzubeugen. Zahlreiche Umwelteinflüsse wie Bakterien, öllösliche Partikel und Schmutz beeinflussen unsere Haut tagtäglich und schwächen ihren natürlichen Säureschutzmantel. Reinigungsprodukte mit Mizellen-Technologie eignen sich opti- mal für eine intensive Gesichtsreinigung, ohne die Haut unnötig zu beanspruchen.

Mizellen sind mikroskopisch kleine Tensidmoleküle aus der Nanotechnologie, die aus einem fett- und einem wasseraffinen Teil bestehen. Sie ummanteln Makeup-Öle sowie -Fette und lösen sie ideal von der Haut.
Louis Widmers neue, auf Tensiden basierende 3-in-1 Mizellen Reinigungslotion vereint drei Produkte in einem: Make-up-Entferner, Tonique und Reinigungsmilch bzw. Waschgel. Dies führt zu einer sehr einfachen, schonenden und dennoch sehr wirkungsvollen Anwendung, die dafür sorgt, dass die Haut aktive Inhaltsstoffe der Pflegeprodukte besser aufnehmen kann. Die neue Mizellen-Reinigungslotion ist besonders für Kontaktlinsenträgerinnen mit sensibler Augenpartie geeignet. Sie ist ausschließlich unparfümiert erhältlich.

Nach Informationen von Louis Widmer 